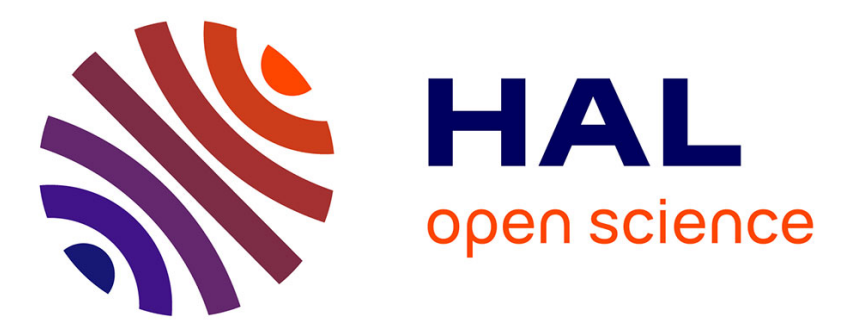

\title{
Charge transport in hybrid platinum/molecule/graphene single molecule junctions
}

Chunhui He, Qian Zhang, Tingwei Gao, Chenguang Liu, Zhenyu Chen, Cezhou Zhao, Chun Zhao, Richard J Nichols, Yannick J Dappe, Li Yang

\section{To cite this version:}

Chunhui He, Qian Zhang, Tingwei Gao, Chenguang Liu, Zhenyu Chen, et al.. Charge transport in hybrid platinum/molecule/graphene single molecule junctions. Physical Chemistry Chemical Physics, 2020, 22 (24), pp.13498 - 13504. 10.1039/d0cp01774d . hal-03032395

\section{HAL Id: hal-03032395 \\ https://hal.science/hal-03032395}

Submitted on 6 Aug 2021

HAL is a multi-disciplinary open access archive for the deposit and dissemination of scientific research documents, whether they are published or not. The documents may come from teaching and research institutions in France or abroad, or from public or private research centers.
L'archive ouverte pluridisciplinaire HAL, est destinée au dépôt et à la diffusion de documents scientifiques de niveau recherche, publiés ou non, émanant des établissements d'enseignement et de recherche français ou étrangers, des laboratoires publics ou privés. 


\title{
Charge Transport in Hybrid Platinum/Molecule/Graphene Single Molecule Junctions
}

Received 00th January 20xx, Accepted 00th January 20xx DOI: $10.1039 / x 0 x \times 00000 x$

\author{
Chunhui He, ${ }^{a, b}$ Qian Zhang, ${ }^{a, b}$ Tingwei Gao, ${ }^{a, b}$ Chenguang Liu, ${ }^{c}$ Zhenyu Chen, ${ }^{b}$ Cezhou Zhao, ${ }^{c}$ Chun \\ Zhao, ${ }^{c}$ Richard J. Nichols, ${ }^{b}$ Yannick J. Dappe, ${ }^{* d}$ Li Yang ${ }^{* a, b}$
}

\begin{abstract}
The single molecule conductance of hybrid platinum/alkanedithiol/graphene junctions has been investigated with a focus on understanding the influence of employing two very different contact types. We call this an "anti-symmetric" configuration, with the two different contacts here being platinum and graphene, which respectively provide very different electronic coupling to the alkanedithiol bridge. Conductance of these junctions is experimentally investigated using by using a non-contact scanning tunnelling microscope (STM) based method called the $I(s)$ technique. These experimental determinations are supported by density functional theory (DFT) calculation. These alkanedithiol bridging molecules conduct electrical current through the highest occupied molecular orbital (HOMO), and junctions formed with Pt/graphene electrode pairs are slightly more conductive than those formed with Au/graphene electrodes which we previously investigated. This is consistent with the lower work function of gold compared to platinum. The measured conductance decays exponentially with the length of the molecular bridge with a low tunnelling decay constant, that has a similar value for Pt/graphene and $\mathrm{Au} / g r a p h e n e$ electrode pairs, respectively. These new results underline the importance of the coupling asymmetry between the two electrodes, more than the type of the metal electrode itself. Importantly, the tunnelling decay constant is much lower than that of alkanedithiols with the symmetrical equivalent, i.e. identical metal electrodes. We attribute this difference to the relatively weak van der Waals coupling at the graphene interface and the strong bond dipole at the Pt-S interface, yielding a decrease in the potential barrier at the interface.
\end{abstract}

\section{Introduction}

Since Aviram and Ratner originally proposed the imaginative concept of using single molecule as a molecular rectifier in 1974, ${ }^{1}$ there have been huge significant developments in molecular electronics. These have included advances in understanding charge transport mechanisms in molecular junctions (MJs), ${ }^{2}$ demonstration of how current across single molecules can be switched using electrochemistry or light, ${ }^{3,4}$ comprehension of the effects of environment and inter-molecular interactions on molecular conductance $^{5}$ and exploitation of quantum interferences in molecular junctions. ${ }^{6} \mathrm{~A}$ recent exciting development has been a commercialization of large area molecular junctions, which have been deployed as the central component in audio distortion circuits

\footnotetext{
a. Department of Chemistry, Xi'an-Jiaotong Liverpool University, 215123, Suzhou, China.E-mail: li.yang@xitlu.edu.cn

b. Department of Chemistry, University of Liverpool, Liverpool, L69 7ZD, UK. c. Department of Electrical and Electronic Engineering, Xi'an-Jiaotong Liverpool University, 215123, Suzhou, China

d.SPEC, CEA, CNRS, Université Paris-Saclay, CEA Saclay 91191 Gif-sur-Yvette Cedex, France. Email : yannick.dappe@cea.fr
}

for guitar pedals. ${ }^{7}$ These, any many other advances continue to play an important role in determining the future development of the field of molecular electronics.

A key factor for integrating molecules into large scale devices is achieving reliable and reproducible electrode-molecule-electrode junctions. Indeed, these so-called molecular junctions (MJs) are a

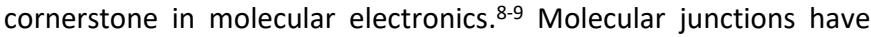
also been downsized to the single-molecule level which represents the ultimate miniaturization limit for molecular devices. The majority of recent studies of single-molecule junctions have featured a "symmetrical" configuration. Such symmetrical configurations use the same electrode contact ( $\mathrm{Au}, \mathrm{Cu}, \mathrm{Ag}$, and Pt etc.) on each side of the molecular bridge, alongside a symmetrical molecular core and the same anchoring groups (thiol, amine, carboxylic acid, methyl sulfide etc.) at each end of the junction. ${ }^{10-14}$ These studies have formed the classical testbed for understanding electron transport through molecular nano-junctions. However, breaking the symmetry of electrodes, molecular wire or anchoring groups can shift the orbital energies, change the distribution of the electronic states, and consequently generate new transport properties of MJs including rectification, molecular switching or negative differential resistance (NDR). It is pertinent to note here that the seminal publication of the 
molecular rectifier concept innovated by Aviram and Ratner feature such a non-symmetrical contact.

A number of studies have shown that breaking the symmetry of molecular junctions can impact the electrical response. Tamaki and co-worker ${ }^{15}$ have reviewed and summarized various junction devices featuring a series of non-symmetric molecular structures and their underlying mechanism of rectification, NDR and switching. Recently charge transport was studied in highly non-symmetric molecules containing a 9'9-spirobifluorene tripodal platform. ${ }^{16}$ The results showed that by making several contacts between the molecule and the electrode, the number of possible molecular junction configurations was reduced and the most apparent charge transport pathway was better defined. MJs containing non-symmetric dipyrimidinyl-diphenyl diblock molecules bridged between two gold electrodes were reported to achieve pronounced rectification behavior. These non-symmetric molecules featured larger currents than their symmetrical counterpart, and their studies highlighted the importance of the molecule-electrode coupling on achieving rectification. ${ }^{17} A$ systematic study has been undertaken with the configuration A-bridge- $A, A$-bridge- $B$ and $B$-bridge- $B$ ( $A=$ thiol terminus and $B=C O O H)$. This study found that when symmetry is broken by using such non-identical anchoring groups (A-bridge-B) the single molecule conductance is smaller when compared with symmetrical molecules. ${ }^{18} \mathrm{Kim}$ and co-workers introduced a hybrid device structure with the amine-terminated oligophenyls sandwiched between a gold tip and a graphite substrate. Rectification behaviour was observed in these junctions and is directly explained by an asymmetry in the electronic coupling to the two contacting electrodes. ${ }^{19}$

The use of non-identical contacting groups at each end of a molecular bridge clearly introduces electronic coupling differences at each contact which has been discussed in previous literature for sandwich type junctions featuring self-assembled monolayers. ${ }^{20}$ Influential factors here include the contact resistance at the molecule-electrode interface, the barrier height for the electron transfer, the alignment at the interfaces of the lowest unoccupied molecular orbital (LUMO) and the highest occupied molecular orbital (HOMO) and the Fermi levels $\left(E_{F}\right)$ of the contacting electrodes. ${ }^{21}$ To date, less concrete data exist in the literature regarding non-symmetric molecule-electrode contacting, where the length dependence is addressed in terms of type of chemical bonding at the contacts (i.e. physisorbed versus chemisorbed). In our earlier studies, we have shown for nonsymmetric hybrid Au-molecule-graphene junctions that the tunneling decay constant $\left(\boldsymbol{\beta}_{\boldsymbol{n}}\right)$ is lower than the corresponding goldmolecule-gold junctions which are by contrast symmetric. For these studies, symmetry breaking was achieved with the contacting (Au/graphene electrode) and different anchoring groups were employed at each ends of the molecular bridge (dithiol, 22,23 diamine, 24 and dicarboxylic acid ${ }^{25}$ ).

The present report extends our study to Pt/alkanedithiol/graphene non-symmetric junctions and highlights the attractive features of this pronounced symmetry breaking achieved through a physisorbed contact at the graphene interface and a chemisorbed contact (Pt-S) at the platinum side. The MJs formed by Pt electrodes have shown higher conductance compared to the equivalent junctions with $\mathrm{Au}$ electrodes. For example, Kiguchi et al. made the direct binding of benzene molecules to two Pt electrodes and the relevant junctions revealed 1 order larger conductance than the equivalent junctions featuring Au-Au electrode pairs. ${ }^{26}$ Ko et al. compared the electronic coupling of thiolate on $\mathrm{Au}, \mathrm{Pd}$ and $\mathrm{Pt}$ electrodes. Their results showed that junctions with $\mathrm{Pt}$ electrodes exhibit about 3.5 fold greater conductance than those of Au electrodes. ${ }^{27}$ In line with these findings our junctions with non-symmetric Pt/graphene contacting also yields a higher conductance at longer lengths and features low decay constants, when compared to similar MJs with $\mathrm{Au} / \mathrm{Au}$ and $\mathrm{Pt} / \mathrm{Pt}$ electrodes. We attribute these findings to the pronounced coupling differences to the non-equivalent electrodes at either end of the molecular bridge. These conclusions are supported by detailed density functional theory (DFT) calculations.

\section{Experimental methods}

Materials. All chemicals were reagent grade and were used as received. All solutions were prepared in mesitylene (99\%, Aladdin). Ethanedithiol (C2), butanedithiol (C4), hexanedithiol (C6), octanedithiol (C8), decanedithiol (C10) were purchased from Alfa Aesar. $0.25 \mathrm{~mm}$ platinum wire (99.99\%) was purchased from Tianjing Lucheng Metal. A ready-to-use fewlayer graphene $(10 \mathrm{~mm} \times 10 \mathrm{~mm})$ on top of a nickel layer was purchased from the Graphene Supermarket, US.

Tip preparation. Pt tips were prepared using electrochemistry etching previously reported. ${ }^{28}$ Briefly, the procedure was divided into two steps. In the first step, the $\mathrm{Pt}$ wire was ultrasonically cleaned in acetone for 10 mins. Then a piece of $\sim 15 \mathrm{~mm}$ long Pt wire was cut and immersed into a calcium chloride electrolyte with a concentration of $30 \mathrm{wt}$. \%. The amount of wire immersed into the solution was approximately 2-3 $\mathrm{mm}$. An AC voltage of $35 \mathrm{~V}$ was applied between the platinum and graphite counter electrode. After $90 \mathrm{~s}$, for the second stage the voltage was then reduced to $18 \mathrm{~V}$ for fine etching in order to sharpen the tips. The etching reaction stopped automatically when the platinum wire section immersed the electrolyte broke off.

Conductance measurement. The compounds under study were prepared at $1 \mathrm{mM}$ concentrations in mesitylene solutions and 1-2 drops of the solution was placed in a STM fluid cell. The graphene substrate was fixed onto the sample plate with silver gel and dried overnight to facilitate good electrical contact plate. The conductance of single molecular junctions was measured using the scanning tunnelling microscopy (STM) $I(s)$ method ( $I=$ current, $s=$ vertical distance), following the method described by Haiss et al. with appropriate modifications to our Bruker STM instrumentation. ${ }^{29}$ Figure 1 shows the schematic diagram of the experimental setup. In the $I(s)$ method the STM tip suffers no direct contact with the substrate, and hence damage to either the tip or the graphene substrate is obviated. The Pt tip was approached to be within electron tunnelling distance of the substrate. During this approach, when the separation was sufficiently close the preset threshold of the setpoint current was attained, and the feedback loop was then disconnected. The STM tip was then rapidly retracted vertically to the initial distance by around $4 \mathrm{~nm}$. This process of extending and retracting the STM tip was repeated many times to find the characteristic plateaus in the recorded current-distance curves, which indicate the successful formation of MJs. The STM-I(s) 
measurements were made at a current setpoint of $1 \mathrm{nA}$ and the ramp rate of $1.03 \mathrm{~Hz}$. The tip bias for all the experiments was set at $\mathrm{V}_{\text {bias }}=+0.3 \mathrm{~V}$ and typically over $10000 /(s)$ curves were gathered. More than 500 curves showing well-defined plateaus and low noise were selected for the statistical analysis. According to the position of the plateaus, the selected curves were segmented into 8 bins equally based on the current set point. The curves from bins with the largest and the second largest numbers were then converted into conductance values to obtain a one-dimensional (1D) electrical conductance histogram. A Gaussian fit was applied to the most dominant peak and the representative conductance value was obtained.

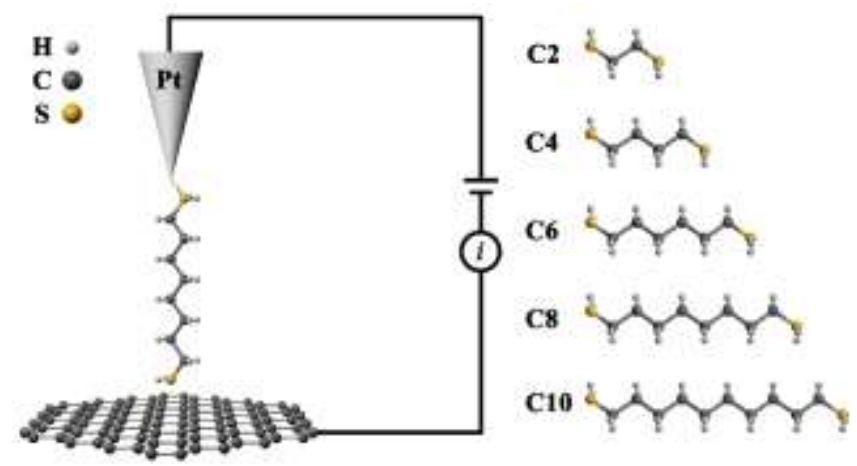

Figure 1. Schematic illustration of forming a $\mathrm{Pt} /$ alkanedithiol/graphene hybrid molecular junction.

Theoretical calculation. Density Functional Theory (DFT) has been used to model the molecular junctions and to determine the electronic transport properties. We have used the DFT code Fireball, which has very efficient localized-orbitals basis sets. ${ }^{30}$ Basis sets of $\mathrm{sp}^{3} \mathrm{~d}^{5}$ numerical orbitals for $\mathrm{Pt}, \mathrm{sp}^{3}$ for $\mathrm{C}$ and $\mathrm{S}$, and $\mathrm{S}$ for $\mathrm{H}$ have been used to determine the atomic configuration and the electronic properties of the junctions. The corresponding cut-off radii (in atomic units) are $s=4.6, p=5.8$ $\mathrm{d}=4.2(\mathrm{Pt}),{ }^{31} \mathrm{~s}=4.5, \mathrm{p}=4.5(\mathrm{C}), \mathrm{s}=3.1, \mathrm{p}=3.9(\mathrm{~S})$, and $\mathrm{s}=4.1$ (H). ${ }^{32}$ Following the procedure developed for the study of gold tips, ${ }^{22}$ we have used a Pt tip of 35 atoms, and a $5 \times 5$ graphene supercell in the $X Y$ plane. Fireball was then used to optimize all the configurations until the forces went under $0.05 \mathrm{eV} / \AA ̊ \AA$. Also, we have employed a Keldysh-Green formalism to compute the conductance of the molecular junctions at $0 \mathrm{~K}$ and zero bias. ${ }^{22}$ 33

\section{Results and discussion}

To study the influence of the tip materials to the hybrid graphene molecular junctions, simple alkanedithiols with their saturated carbon atom chains (polymethylene bridge) have been used as model compounds. Polymethylene chains are amongst the most popular choices of model molecular bridges for investigating the molecule-electrode coupling and junction conductance. ${ }^{18,22,23}$ Pertinent features include their stable chemical properties, alongside the large gap (several electron volts) between the HOMO and LUMO. In this work, the conductance measurements were performed for ethanedithiol (C2), butanedithiol (C4), hexanedithiol (C6), octanedithiol (C8), and decanedithiol (C10) molecular junctions. Figure 2 shows typical $I(s)$ curves measured for all the molecular junctions in a liquid cell filled with pure mesitylene solvent. The formation of the molecular junction is readily identified in each curve by a characteristic plateau, followed by a sudden decay. In the absence of a molecule between the two electrodes, the conductance curve simply decays exponentially with no visible step observed (not shown here). In Figure 2, it is clear that for the junctions studied here, the conductance values are strongly dependent on the length of the molecular backbone. As such, for bridges with smaller numbers of methylene groups, the conductance is higher. For example, the conductance of the longer Pt/C6/Graphene junctions is located around 25-40 nS, while, by contrast, the conductance of the Pt/C2/Graphene junctions which are shorter falls around 85-100 nS. Notice that the plateau height shows some variation for the same molecular junction, indicating the stochastic nature of the junction formation and breaking events. To better illustrate the predominant molecular junction configuration, a large number of molecular junctions are repeatedly generated and a statistical analysis from at least $500 /(s)$ curves is applied to all the tested molecules. The plotted 1D histograms are shown in Figure 3 and a progression of conductance of $1, n$-alkanedithiol $(n=2,4,6,8,10)$ with molecular length is observed. Only a pronounced main peak was found for each histogram. After a Gaussian fit, the resulting conductance values of C2 (black), C4 (purple), C6 (red), C8 (green) and C10 (blue) are 99, 45, 29, 18, and $7.5 \mathrm{nS}$, respectively. The conductance values decrease with increasing molecular length, as is expected. Interestingly, the conductance values have a certain spread especially for shorter molecules, and this distribution is generally proportional to the magnitude of the experimental value. Shorter junctions present higher conductance values, so their relative distribution of conductance values is normally greater than their longer homologues. For example, Pt/C8/graphene junctions located between $40 \mathrm{nS}$ to $70 \mathrm{nS}$, while $\mathrm{Pt} / \mathrm{C} 10 /$ graphene junctions located between $5 \mathrm{nS}$ to $10 \mathrm{nS}$.

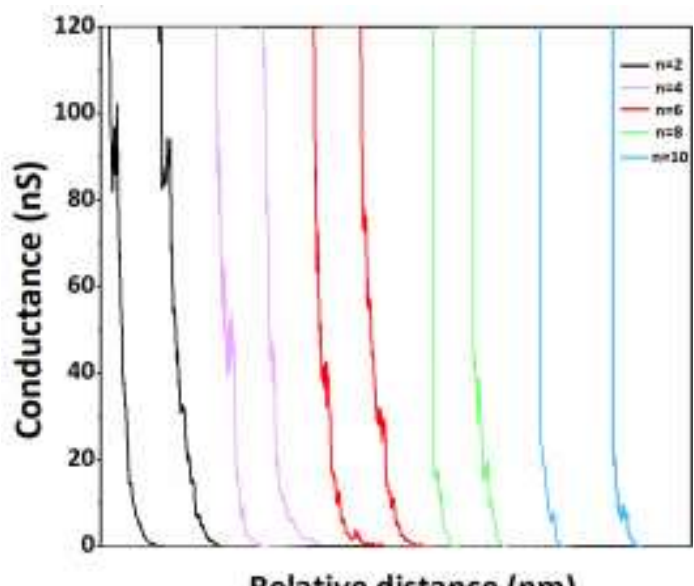

Figure 2. Typical $I(s)$ curves of $\mathrm{Pt} / \mathrm{S}-\left(\mathrm{CH}_{2}\right)_{\mathrm{n}}-\mathrm{SH} / \mathrm{graphene}$ molecular junctions, with different alkanedithiol length. These are $n=2$ (black solid line), 4 (purple lines), 6 (red lines), 8 (light green lines) and 10 (light blue lines). 


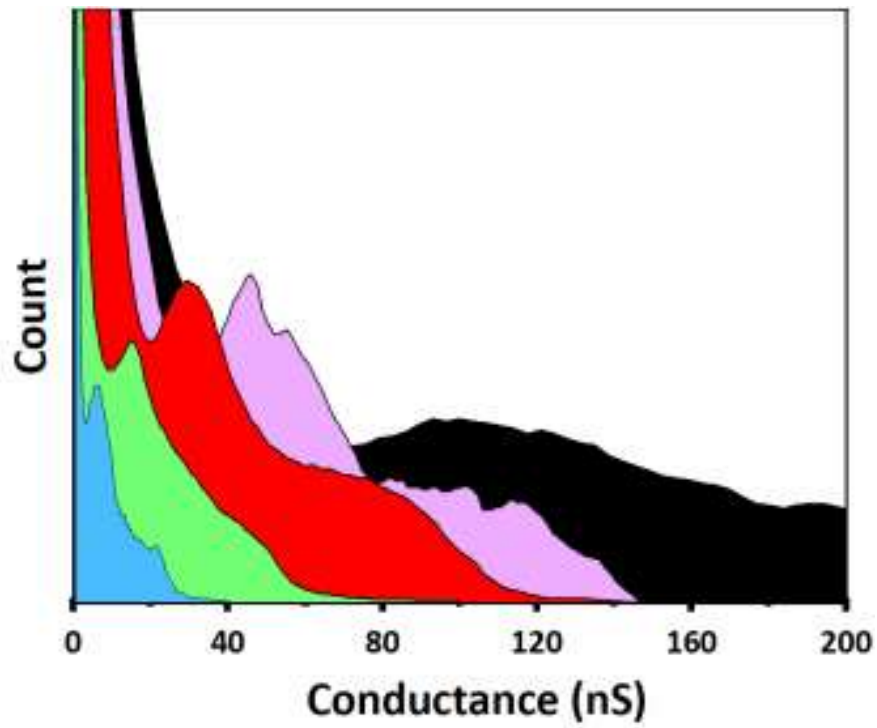

Figure 3. 1D histograms for the conductance of Pt/S- $\left(\mathrm{CH}_{2}\right) \mathrm{n}$ $\mathrm{SH} / g r a p h e n e$ molecular junctions with different alkanedithiol length: $\mathrm{n}=2$ (filled solid black area), 4 (purple area), 6 (red area), 8 (light green area) and 10 (light blue area). The coloring is consistent with that used in figure 2 .

The conductance of alkanedithiol bound between graphene and Pt contacts is consistent with our previous report, ${ }^{22}$ where we used graphene and $\mathrm{Au}$ electrodes, which established the reliability of the approach and the reproducible results obtained when using graphene as a bottom electrode. Figure 4 shows a comparative analysis of the length dependence of the electrical conductance of $\mathrm{Pt} / \mathrm{S}-\left(\mathrm{CH}_{2}\right)_{\mathrm{n}}-\mathrm{SH} / \mathrm{graphene}$ (yellow) and $\mathrm{Au} / \mathrm{S}-$ $\left(\mathrm{CH}_{2}\right)_{n}$-SH/graphene (blue) junctions, respectively. Here the natural logarithm of the molecular conductance is plotted as a function of the molecular length expressed in the number of methylene groups. The corresponding theoretical results are also presented for $\mathrm{Pt} / \mathrm{S}-\left(\mathrm{CH}_{2}\right)_{\mathrm{n}}-\mathrm{SH} /$ graphene (red, theory) and $\mathrm{Au} / \mathrm{S}-\left(\mathrm{CH}_{2}\right)_{n}-\mathrm{SH} /$ graphene (green, theory) junctions respectively. The theoretical conductance of the Pt molecular junctions at the Fermi level is extracted from the electronic transmission plot shown in Figure 5, where $\boldsymbol{G}=\boldsymbol{T}\left(\boldsymbol{E}_{\boldsymbol{F}}\right)$. One can clearly notice the proximity of the HOMO level to the Fermi level of the Pt electrodes. Consequently, as in the case of the Augraphene electrodes, the charge transport in the junction is dominated by the HOMO level.

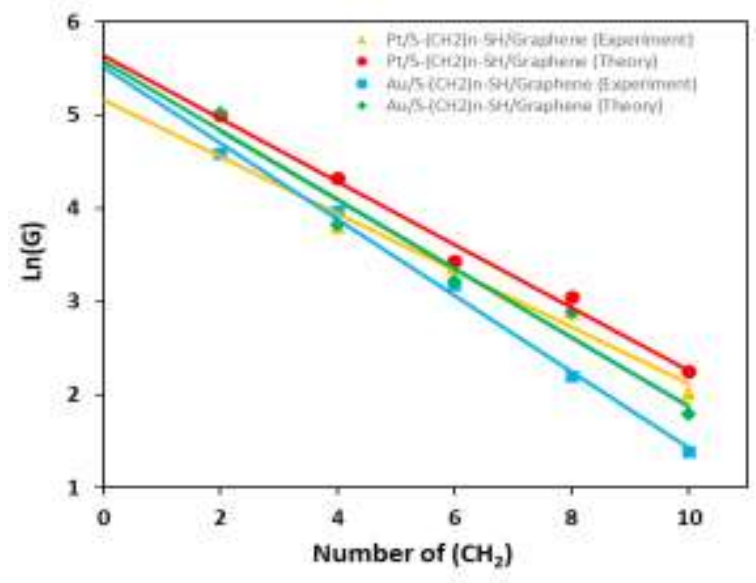

Figure 4. Plots of the natural logarithm of the conductance as a function of junction length for the following systems: Pt/S$\left(\mathrm{CH}_{2}\right)_{n}$-S/graphene (experiment, yellow), Pt/S- $\left(\mathrm{CH}_{2}\right)_{\mathrm{n}^{-}}$ $\mathrm{S} /$ graphene (theory, red), $\mathrm{Au} / \mathrm{S}-\left(\mathrm{CH}_{2}\right)_{\mathrm{n}}-\mathrm{S} /$ graphene (experiment, blue) and $\mathrm{Pt} / \mathrm{S}-\left(\mathrm{CH}_{2}\right)_{n}-\mathrm{S} /$ graphene (theory, green). The length is expressed as the number of methylene groups in the alkanedithiol molecular bridge.

a)
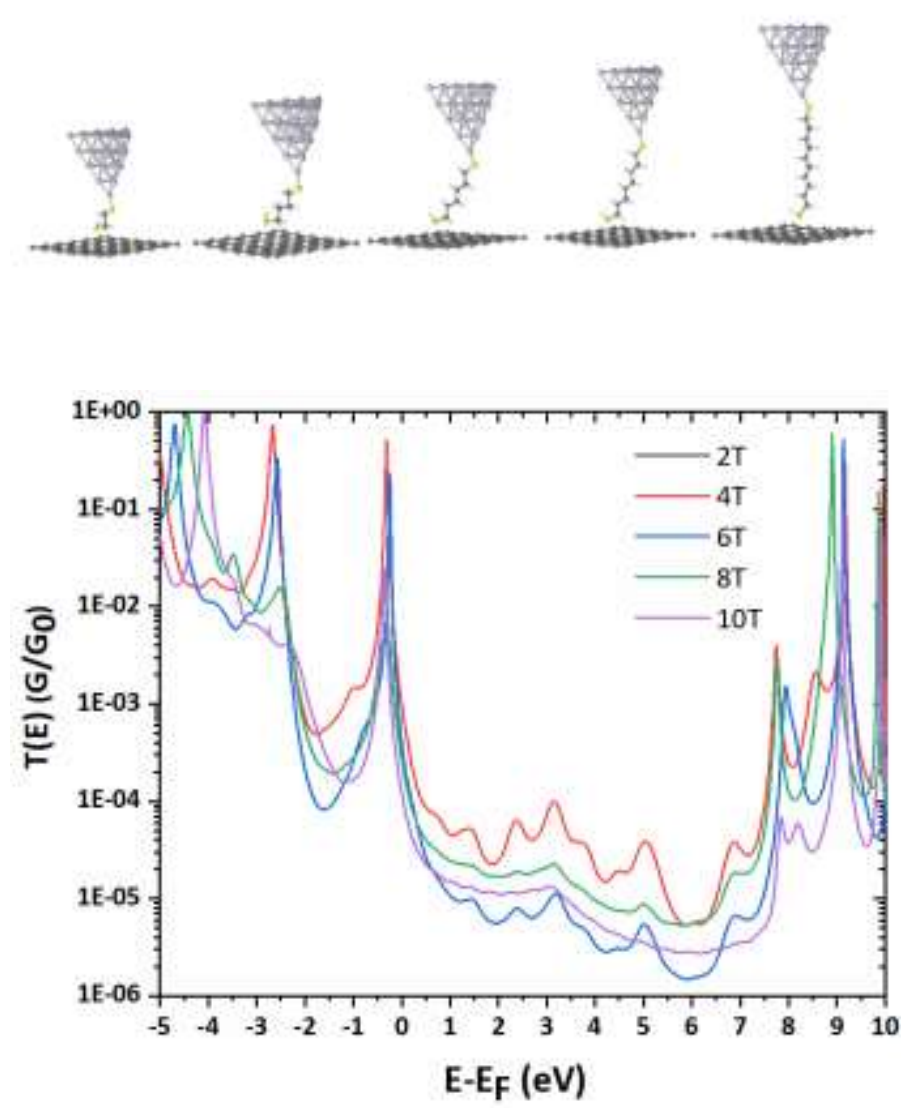

Figure 5. a) Atomic configurations of the different molecular junctions used for DFT calculations and b) corresponding calculated transmissions for Pt/S- $\left(\mathrm{CH}_{2}\right) \mathrm{n}-\mathrm{SH} / \mathrm{graphene}$ molecular junctions $(n=2,4,6,8,10)$. 
As expected, the measured conductance decreases with the molecular length for both Pt and Au tips when forming nonsymmetric molecular junctions with graphene as the other contact. The conductance values measured for the two junctions types (Pt/S- $\left(\mathrm{CH}_{2}\right)_{n}-\mathrm{SH} / \mathrm{graphene}$ and $\mathrm{Au} / \mathrm{S}-\left(\mathrm{CH}_{2}\right)_{\mathrm{n}^{-}}$ $\mathrm{SH} /$ graphene) are similar. Although the individual theoretical conductance value is slightly overestimated (well-known overestimation of DFT calculations ${ }^{34-36}$ ), the overall simulation results are in good agreement with the experimental conductance values. A linear fit to the conductance points and the slope of the plot of $\ln (G)$ versus the number of $-\mathrm{CH}_{2}$ units gives the value of the attenuation factor $\boldsymbol{\beta}_{\boldsymbol{n}}$, since the conductance can be expressed as

$$
G=A \exp \left(-\beta_{n} N\right)
$$

where $G$ is the conductance, $N$ is the number of methylene groups in the alkane chain and $A$ is a constant inversely proportional to the contact resistance between the molecular bridge and the contacting electrode. Noted from this expression, a low value of $\boldsymbol{\beta}_{\boldsymbol{n}}$ indicates a slow conductance decay, leading to a more effective electronic transport at longer lengths. In agreement with our previous results ${ }^{22-25}$, molecular junctions with $\mathrm{Pt} / \mathrm{graphene}$ electrodes result in a low decay constant when compared to the analogous MJs with $\mathrm{Pt} / \mathrm{Pt}$ contacting electrodes. These leads to a higher conductance for MJs with $\mathrm{N}>6$ with Pt/graphene contacts, than those achieved for either $\mathrm{Au} / \mathrm{Au}$ or Pt/Pt contacting.

Table 1 collects together the conductance and $\boldsymbol{\beta}_{\boldsymbol{n}}$ values of the MJs $\left(\mathrm{SH}-\left(\mathrm{CH}_{2}\right)_{n}-\mathrm{SH}\right)$ with Pt/graphene electrodes, Au/graphene electrodes, and $\mathrm{Au} / \mathrm{Au}$ electrodes. This summary shows that the decay constant $(0.30)$ of $\mathrm{Pt} / \mathrm{S}-\left(\mathrm{CH}_{2}\right)_{\mathrm{n}}-\mathrm{SH} / \mathrm{graphene}$ junctions is less than that of the $\mathrm{Au} / \mathrm{S}-\left(\mathrm{CH}_{2}\right)_{\mathrm{n}}-\mathrm{SH} / \mathrm{Au}$ junctions (1.08) and also less than Pt/S- $\left(\mathrm{CH}_{2}\right)_{n}-\mathrm{S} / \mathrm{Pt}$ junctions (1.05). ${ }^{27}$ This significantly smaller value of $\boldsymbol{\beta}_{\boldsymbol{n}}$ for $\mathrm{Pt} / \mathrm{graphene}$ junctions is similar to the $\mathrm{Au} / \mathrm{S}-\left(\mathrm{CH}_{2}\right)_{\mathrm{n}}-\mathrm{SH} / \mathrm{graphene}$ junctions (0.41) and both also are in good agreement with the theoretical computations of the $\boldsymbol{\beta}_{n}$ values. This smaller value is attributed to the symmetry breaking induced when using graphene as the second electrode. Indeed, the electrostatics of the molecular junction is unbalanced between the strong dipole at the Pt-S interface and the weak dipole at the graphene-SH interface. ${ }^{22}$ Indeed, the alkanedithiol is physisorbed on graphene, leading to no orbital hybridization between the $\mathrm{SH}$ anchoring group and graphene, in contrast to the Au-S interface. This is confirmed through DFT calculations and contact resistance measurements. This results in a shift of the HOMO level toward the Fermi level, with respect to a standard Pt-Pt junction, which reduces the electronic barrier at the interface and consequently the attenuation factor. This is exactly the same mechanism as for the Augraphene electrodes. ${ }^{22}$ Hence, this behaviour is not dependent on the metal used for the electrodes, but more on the coupling difference, strong at the metal/S interface and weak at the graphene/SH interface.

Table 1. Experimental and theoretical conductance values for the following molecular junction systems: Pt/S- $\left(\mathrm{CH}_{2}\right)_{n}-\mathrm{S} /$ graphene, $\mathrm{Au} / \mathrm{S}$ -
$\left(\mathrm{CH}_{2}\right)_{n}$-S/graphene and $\mathrm{Au} / \mathrm{S}-\left(\mathrm{CH}_{2}\right)_{n}-\mathrm{S} / \mathrm{Au}$ molecular junctions. Tunneling decay constant $\left(\boldsymbol{\beta}_{n}\right)$ are tabulated on the right-hand side.

\begin{tabular}{|c|c|c|c|c|c|}
\hline \multirow{2}{*}{$\begin{array}{l}\text { Molecular } \\
\text { junctions }\end{array}$} & \multirow{2}{*}{$\begin{array}{l}\text { Number } \\
\text { of } \mathrm{CH}_{2}\end{array}$} & \multicolumn{2}{|c|}{ Conductance (nS) } & \multicolumn{2}{|c|}{ Decay constant $\left(\beta_{n}\right)$} \\
\hline & & Experiment & Theory & Experiment & Theory \\
\hline $\mathrm{Pt} / \mathrm{S}-\left(\mathrm{CH}_{2}\right)_{\mathrm{n}^{-}}$ & 2 & 99 & 148 & & \\
\hline \multirow[t]{4}{*}{ S/graphene } & 4 & 45 & 75 & & \\
\hline & 6 & 29 & 31 & 0.30 & 0.34 \\
\hline & 8 & 18 & 21 & & \\
\hline & 10 & 7.5 & 9.5 & & \\
\hline $\mathrm{Au} / \mathrm{S}$ & 2 & 98 & 152 & & \\
\hline$\left(\mathrm{CH}_{2}\right)_{\mathrm{n}^{-}}$ & 4 & 53 & 46 & & \\
\hline S/graphene & 6 & 24 & 25 & 0.41 & 0.37 \\
\hline \multirow[t]{2}{*}{22} & 8 & 9 & 18 & & \\
\hline & 10 & 4 & 6 & & \\
\hline \multirow{3}{*}{$\begin{array}{l}\mathrm{Au} / \mathrm{S}- \\
\left(\mathrm{CH}_{2}\right)_{n}-\mathrm{S} / \mathrm{Au} \\
35\end{array}$} & 6 & 28.2 & & 1.08 & \\
\hline & 8 & 3.9 & & & \\
\hline & 10 & 0.2 & & & \\
\hline \multirow{3}{*}{$\begin{array}{l}\mathrm{Pt} / \mathrm{S}-\left(\mathrm{CH}_{2}\right)_{\mathrm{n}}- \\
\mathrm{S} / \mathrm{Pt}^{27}\end{array}$} & 6 & 30.2 & 341.0 & 1.05 & \\
\hline & 8 & 6.6 & 65.8 & & \\
\hline & 10 & 0.6 & 11.7 & & \\
\hline
\end{tabular}

For further comparison, the findings from our earlier study on carbon fibre/S- $\left(\mathrm{CH}_{2}\right)_{n}-\mathrm{SH} /$ graphene junctions are discussed next. ${ }^{37}$ As discussed previously carbon fibre "behaves like a gold tip" for such junctions, leading to a similar attenuation factor (0.38), but also a slightly reduced conductance. Again, the conductance depends dramatically on the symmetry breaking and the weak coupling between the molecule and the carbon fiber electrode. Overall, we find no significant difference for the value of $\boldsymbol{\beta}_{\boldsymbol{n}}$ for all graphene hybrid molecular junctions using $\mathrm{Pt}, \mathrm{Au}$ or carbon fiber tips. In the same manner, Engelkes et $a^{38}$ obtained no notable differences in $\boldsymbol{\beta}_{\boldsymbol{n}}$ for 18 different symmetric junction types employing three metals ( $\mathrm{Pt}, \mathrm{Au}$, or $\mathrm{Ag}$ ) for the substrate and tip, with alkanethiols and alkanedithiols as bridging molecules. The previously reported study ${ }^{39}$ involving a series of amine-terminated oligophenyls and polymethylene (alkane) bridges also shows a higher conductance with gold electrodes than with silver. In that study $\boldsymbol{\beta}_{\boldsymbol{n}}$ is generally independent of whether the contacts are $\mathrm{Au}$ or $\mathrm{Ag}$.

The fits in Figure 4 can be extended to zero length, with the resulting intercept of those plots giving the contact resistances $\left(R_{0}\right)$. For Pt/S$\left(\mathrm{CH}_{2}\right)_{\mathrm{n}}$-SH/graphene junctions, the contact resistance is $5.8 \mathrm{M} \Omega$, which is slightly larger than that obtained for $\mathrm{Au} / \mathrm{S}-\left(\mathrm{CH}_{2}\right)_{\mathrm{n}}$ $\mathrm{SH} /$ graphene junctions $\left(3.9 \mathrm{M} \Omega\right.$ ) in our previous study ${ }^{22}$ and much higher than that obtained for $\mathrm{Au} / \mathrm{Au}$ symmetric contacts processing two Au-S-alkane contacts $(27 \mathrm{k} \Omega)$. This difference points to the electronic coupling between molecular bridge and electrodes being significantly weaker at the graphene-molecule interface. At the other electrode there is a large bond dipole associated with the metal-S contact resulting from the electron donation from the sulfur to Pt. A study reported by Kim et al ${ }^{19}$ also found that the $R_{0}$ is three orders of magnitude higher for $\mathrm{Au} /$ molecule/graphite junctions than that of $\mathrm{Au} / \mathrm{molecule/Au}$ junctions. They proposed a weak electronic coupling at molecule-graphite contact. Also, in agreement with previous results, the bond dipole is profoundly dependent on the metal type for alkanedithiols, ${ }^{40}$ namely the bond dipole increases with the work function. The work function for Pt is $5.65 \mathrm{eV}$ and 5.20 
$\mathrm{eV}$ for $\mathrm{Au}$, which self-consistently explains the behaviour in terms of the junction transport characteristics.

\section{Conclusions}

We have experimentally determined the electrical conductance and tunnelling decay constant in $\mathrm{Pt} / \mathrm{S}-\left(\mathrm{CH}_{2}\right) \mathrm{n}-\mathrm{SH} / \mathrm{graphene}$ molecular junctions using the STM $I(s)$ method. The conductance of $\mathrm{Pt} /$ graphene molecular junctions decreases exponentially with the increase of the molecular length and is higher than equivalent $\mathrm{Pt} / \mathrm{Pt}$ junctions for longer junctions. Importantly, the tunnelling decay constant of about 0.30 is much lower than that of symmetric metal electrode. configurations. This is in good agreement with previous studies for polymethylene molecular bridges terminated with either thiol, amine or carboxylic acids, where replacement of a metal bottom electrode with graphene considerably reduces the length attenuation factor of the electrical conductance. These results are confirmed by DFT calculations, which show that the HOMO orbital (which is closer to the Fermi level than the LUMO for these systems) mediates the charge transfer. As a consequence, this study confirms the role of the coupling asymmetry between Pt, Au or even carbon fibre on one side of the molecular bridge and the graphene electrode on the other side. This coupling asymmetry leads to the reduction of the length attenuation factor of the molecular junction current.

\section{Conflicts of interest}

There are no conflicts to declare.

\section{Acknowledgements}

This work was supported by the National Natural Science Foundation of China (NSFC Grants 21503169), Suzhou Industrial Park Initiative Platform Development for Suzhou Municipal Key Lab for New Energy Technology (RR0140), Key Program Special Fund in XJTLU (KSF-E-28) and the XJTLU Research Development Fund (RDF-14-02-42, RDF-16-01-33 and REF-19-01-05)

\section{References}

1. A. Aviram and M. A. Ratner, Chem. Phys. Lett., 1974, 29 277.

2. S. H. Choi, B. Kim and C. D. Frisbie, Science, 2008, 320 1482.

3. C. C. Jia, A. Migliore, N. Xin, S. Y. Huang, J. Y. Wang, Q. Yang, S. P. Wang, H. L. Chen, D. M. Wang, B. Y. Feng, Z. R. Liu, G. Y. Zhang, D. H. Qu, H. Tian, M. A. Ratner, H. Q. Xu, A. Nitzan and X. F. Guo, Science, 2016, 353, 1443.

4. N. J. Kay, S. J. Higgins, J. O. Jeppesen, E. Leary, J. Lycoops, J. Ulstrup and R. J. Nichols, J. Am. Chem. Soc., 2012, 134, 16817.

5. W. Haiss, H. V. Zalinge, D. Bethell, J. Ulstrup, D. J. Schiffrin and R. J. Nichols, Faraday Discuss., 2006, 131, 253.

6. H. L. Chen, H. N. Zheng, C. Hu, K. Cai, Y. Jiao, L. Zhang, F. Jiang, I. Roy, Y. Y. Qiu, D. K. Shen, Y. N. Feng, F. M.
Alsubaie, H. Guo, W. J. Hong and J. F. Stoddart, Matter, 2020, 2, 378.

7. A. J. Bergren, L. Zeer-Wanklyn, M. Semple, N. Pekas, B. Szeto and R. L. Mccreery, J. Phys-Condens. Mat., 2016, 28, 094011

8. T. A. Su, M. Neupane, M. L. Steigerwald, L. Venkataraman and C. Nuckolls, Nat. Rev. Mater., 2016, 1, 16002.

9. R. J. Nichols and S. J. Higgins, Annu. Rev. Anal. Chem., 2015, 8, 389.

10. S. Y. Jang, P. Reddy, A. Majumdar and R. A. Segalman, Nano Lett., 2006, 6, 2362.

11. L. Venkataraman, J. E. Klare, C. Nuckolls, M. S. Hybertsen and M. L. Steigerwald, Nature, 2006, 442, 904.

12. Y. H. Wang, X. Y. Zhou, Y. Y. Sun, D. Han, J. F. Zheng, Z. J. Niu and X. S. Zhou, Electrochim. Acta, 2014, 123, 205.

13. Z. L. Peng, Z. B. Chen, X. Y. Zhou, Y. Y. Sun, J. H. Liang, Z. J. Niu, X. S. Zhou and B. W. Mao, J. Phys. Chem. C, 2012, 116, 21699.

14. Y. H. Wang, D. F. Li, Z. W. Hong, J. H. Liang, D. Han, J. F. Zheng, Z. J. Niu, B. W. Mao and X. S. Zhou, Electrochem. Commun., 2014, 45, 83.

15. T. Tamaki and T. Ogawa, Topics Curr. Chem., 2017, 375, 79.

16. J. Sebera, V. Kolivoska, M. Valasek, J. Gasior, R. Sokolova, G. Meszaros, W. J. Hong, M. Mayor and M. Hromadova, J. Phys. Chem. C, 2017, 121, 12885.

17. I. Diez-Perez, J. Hihath, Y. Lee, L. P. Yu, L. Adamska, M. A. Kozhushner, I. I. Oleynik and N. J. Tao, Nat. Chem., 2009, 1, 635 .

18. S. Martin, D. Z. Manrique, V. M. Garcia-Suarez, W. Haiss, S. J. Higgins, C. J. Lambert and R. J. Nichols, Nanotech., 2009, 20, 125203.

19. T. Kim, Z. Liu, C. Lee, J. B. Neaton and L. Venkataraman, PNAS, 2014, 111, 10928.

20. J. M. Seminario, C. E. De La Cruz and P. A. Derosa, J. Am. Chem. Soc. 2001, 123, 5616.

21. J. M. Beebe, V. B. Engelkes, L. L. Miller and C. D. Frisbie, J. Am. Chem. Soc., 2002, 124, 11268.

22. Q. Zhang, L. L. Liu, S. H. Tao, C. Y. Wang, C. Z. Zhao, C. Gonzalez, Y. J. Dappe, R. J. Nichols and L. Yang, Nano Lett., 2016, 16, 6534.

23. Q. Zhang, S. H. Tao, Y. Q. Fan, C. Zhao, C. Z. Zhao, W.T. Su, Y. J. Dappe, R. J. Nichols and L. Yang, J. Phys. Chem. C, 2018, 122, 23200 .

24. Q. Zhang, S. H. Tao, R. W. Yi, C. H. He, C. Zhao, W. T. Su, A. Smogunov, Y. J. Dappe, R. J. Nichols and L. Yang, J. Phys. Chem. Lett., 2017, 8, 5987.

25. L. L. Liu, Q. Zhang, S. H. Tao, C. Z. Zhao, E. Almutib, Q. AlGaliby, S. W. Bailey, I. Grace, C. J. Lambert, J. Du and L. Yang, Nanoscale, 2016, 8, 14507.

26. M. Kiguchi, O. Tal, S. Wohlthat, F. Pauly, M. Krieger, D. Djukic, J. C. Cuevas and J. M. van Ruitenbeek, Phys. Rev. Lett., 2008, 101, 046801.

27. C. H. Ko, M. J. Huang, M. D. Fu and C. H. Chen, J. Am. Chem. Soc., 2010, 132, 756.

28. Z. Yi and M. Zhang, Rev. Sci. Instrum., 2015, 86, 085105.

29. W. Haiss, H. van Zalinge, S. J. Higgins, D. Bethell, H. Höbenreich, D. J. Schiffrin and R. J. Nichols, J. Am. Chem. Soc., 2003, 125, 15294.

30. J. P. Lewis, P. Jelínek, J. Ortega, A. A. Demkov, D.G. Trabada, B. Haycock, H. Wang, G. Adams, J. K. Tomfohr, E. Abad, H. Wang and D. A. Drabold, Phys. Status Solidi B, 2011, 248, 1989.

31. A. J. Martínez-Galera, N. Nicoara, J. I. Martínez, Y. J. Dappe, J. Ortega and J. M. Gómez-Rodríguez, J. Phys. Chem. C, 2014, 118, 12782 
32. M. A. Basanta, Y. J. Dappe, P. Jelínek and J. Ortega, Comput. Mater. Sci., 2007, 39, 759.

33. C. González, E. Abad, Y. J. Dappe and J. C. Cuevas, Nanotech., 2016, 27, 105201.

34. X. D. Cui, X. Zarate, J. Tomfohr, A. Primak, A. L. Moore, T. A. Moore, D. Gust, G. Harris, O. F. Sankey and S. M. Lindsay, Nanotech., 2002, 13, 5.

35. F. Chen, X. L. Li, J. Hihath, Z. F. Huang, N. J. Tao, . J. Am. Chem. Soc., 2006, 128, 15874.

36. M. Strange, C. Rostgaard, H. Hakkinen and K. S. Thygesen, Phys. Rev. B, 2011, 83, 115108.

37. C. H. He, Q. Zhang, S. H. Tao, Zhao, C. Z. Zhao, W.T. Su, Y. J. Dappe, R. J. Nichols and L. Yang, Phys. Chem. Chem. Phys., 2018, 20, 24553.

38. V. B.; Engelkes, J. M.; Beebe and C. D. Frisbie, J. Am. Chem. Soc., 2004, 126, 14287.

39. T. Kim, H. Vazquez, M. S. Hybertsen and L. Venkataraman, Nano Lett., 2013, 13, 3358.

40. B. Kim, S. H. Choi, X. Y. Zhu and C. D. Frisbie, J. Am. Chem. Soc., 2011, 133, 19864. 